\title{
Predictive biomarkers for death and rehospitalization in comorbid frail elderly heart failure patients
}

Cristina Pacho 1,4, Mar Domingo ${ }^{2}$, Raquel Núñez ${ }^{1}$, Josep Lupón ${ }^{2,4,5}$, Julio Núñez ${ }^{5,6,7}$, Jaume Barallat ${ }^{3}$, Pedro Moliner ${ }^{2}$, Marta de Antonio ${ }^{2}$, Javier Santesmases ${ }^{1,4}$, Germán Cediel ${ }^{2}$, Santiago Roura ${ }^{8}$, M. Cruz Pastor ${ }^{3}$, Jordi Tor ${ }^{1,4}$ and Antoni Bayes-Genis $2,4,58^{*}$

\begin{abstract}
Background: Heart failure (HF) is associated with a high rate of readmissions within 30 days post-discharge and in the following year, especially in frail elderly patients. Biomarker data are scarce in this high-risk population. This study assessed the value of early post-discharge circulating levels of ST2, NT-proBNP, CA125, and hs-Tnl for predicting 30-day and 1-year outcomes in comorbid frail elderly patients with HF with mainly preserved ejection fraction (HFpEF).

Methods: Blood samples were obtained at the first visit shortly after discharge ( $4.9 \pm 2$ days). The primary endpoint was the composite of all-cause mortality or HF-related rehospitalization at 30 days and at 1 year. All-cause mortality alone at one year was also a major endpoint. HF-related rehospitalizations alone were secondary end-points.

Results: From February 2014 to November 2016, 522 consecutive patients attending the STOP-HF Clinic were included ( $57.1 \%$ women, age $82 \pm 8.7$ years, mean Barthel index $70 \pm 25$, mean Charlson comorbidity index $5.6 \pm 2$. 2). The composite endpoint occurred in $8.6 \%$ patients at 30 days and in $38.5 \%$ at 1 year. In multivariable analysis, ST2 [hazard ratio (HR) 1.53; 95\% Cl 1.19-1.97; $p=0.001$ ] was the only predictive biomarker at 30 days; at 1 year, both ST2 (HR 1.34; 95\% Cl 1.15-1.56; $p<0.001)$ and NT-proBNP (HR 1.19; 95\% Cl 1.02-1.40; $p=0.03$ ) remained significant. The addition of ST2 and NT-proBNP into a clinical predictive model increased the AUC from 0.70 to 0.75 at 30 days $(p=0.02)$ and from 0.71 to 0.74 at 1 year $(p<0.05)$. For all-cause death at 1 year, ST2 (HR 1.50; 95\% Cl 1.26-1.80; $p<$ 0.001 ), and CA125 (HR 1.41; 95\% Cl 1.21-1.63; $\mathrm{p}<0.001$ ) remained independent predictors in multivariable analysis. The addition of ST2 and CA125 into a clinical predictive model increased the AUC from 0.74 to $0.78(p=0.03)$. For HF-related hospitalizations, ST2 was the only predictive biomarker in multivariable analyses, both at 30 days and at 1 year.
\end{abstract}

Conclusions: In a comorbid frail elderly population with HFpEF, ST2 outperformed NT-proBNP for predicting the risk of all-cause mortality or HF-related rehospitalization. ST2, a surrogate marker of inflammation and fibrosis, may be a better predictive marker in high-risk HFpEF.

\footnotetext{
* Correspondence: abayesgenis@gmail.com

${ }^{2}$ Servei de Cardiologia i Unitat d'Insuficiència Cardíaca, Hospital Universitari

Germans Trias i Pujol, Badalona, Barcelona, Spain

${ }^{4}$ Department de Medicina, Universitat Autònoma de Barcelona, Barcelona,

Spain

Full list of author information is available at the end of the article
}

(c) The Author(s). 2018 Open Access This article is distributed under the terms of the Creative Commons Attribution 4.0 International License (http://creativecommons.org/licenses/by/4.0/), which permits unrestricted use, distribution, and reproduction in any medium, provided you give appropriate credit to the original author(s) and the source, provide a link to the Creative Commons license, and indicate if changes were made. The Creative Commons Public Domain Dedication waiver (http://creativecommons.org/publicdomain/zero/1.0/) applies to the data made available in this article, unless otherwise stated. 


\section{Background}

Heart failure (HF) is associated with a high rate of readmission within 30 days after discharge $[1,2]$. The rehospitalization rates are as high as $20-30 \%$ in the first month and increase further during the following year [3-6]. This represents a health, social, and economic burden [7-9], especially for elderly patients, as the prevalence of HF is higher in those over 80 years old $[10,11]$. Elderly HF patients differ from younger patients in terms of etiology, comorbidities [12], and phenotype, and elderly patients have a higher prevalence of heart failure with preserved ejection fraction (HFpEF) [13, 14]. This clinical difference impacts short- and mid-term outcomes, and readmissions can be due to exacerbations of the underlying disease [15] as well as to other medical problems [16].

Developing risk prediction models to help in clinical decision making is a major challenge in this elderly HFpEF population [17]. Notably, identifying individuals who are at the highest risk of readmission could lay the groundwork for precision medicine to develop customized preventive measures [18]. Indeed, risk stratification may be refined by the use of biomarkers of different pathophysiologic processes that are not necessarily reflected by established clinical risk factors [19]. Predictive biomarker data in such special populations are scarce and the role of biomarkers in elderly subjects with HF is challenged by the presence of comorbidities [20].

Accordingly, our aim was to assess the value of a panel of 4 biomarkers for predicting 30-day and 1-year outcomes in a population of fragile elderly patients with comorbidities and mainly HFpEF. We analyzed the following biomarkers: N-terminal pro-brain natriuretic peptide (NT-proBNP; a marker of myocardial stretch and neurohormonal activation), interleukin-1 receptorlike 1 (ST2; a marker of inflammation and stretch and extracellular matrix remodeling), high-sensitivity troponin I (hs-TnI; a surrogate marker of myocardial injury), and cancer antigen 125 (CA125; a marker of systemic congestion in $\mathrm{HF}$ ).

\section{Methods}

\section{Study population}

This prospective single-center investigation was performed as part of the STructured multidisciplinary outpatient clinic for Old and frail Post-discharge patients hospitalized for HF (STOP-HF-Clinic) study, which aimed to reduce readmission rates and facilitate the transition to primary care [21]. The STOP-HF-Clinic study included the most vulnerable patients with a primary hospital diagnosis of HF who were admitted for acutely decompensated HF to internal medicine and geriatric wards [22]. The interventions performed in the STOP-HF-Clinic study and at routine visits were reported previously [20]. At the first visit, a mean of $4.9 \pm$
2 days after discharge, the following were obtained from each patient: clinical, demographic, and treatment data; Charlson comorbidity index and Barthel functional score; and a blood sample.

\section{Analytical assays \\ ST2 assay}

ST2 was measured using a high-sensitivity sandwich monoclonal immunoassay (Presage ST2 assay, Critical Diagnostics, San Diego, CA, USA). The hs-ST2 assay had a within-run coefficient of $<2.5 \%$ and a total coefficient of variation of $4 \%$.

\section{NT-proBNP assay}

NT-proBNP was quantified using the AQT90 FLEX immunoassay (Radiometer Medical). According to the manufacturer, there is no detectable cross-reactivity with ANP, BNP, or CNP, and the within-day CV is $<10 \%$ in samples with NT-pro-BNP concentrations higher than $73 \mathrm{pg} / \mathrm{mL}$.

\section{CA125 assay}

Ca125 was measured in an Architect i2000 platform (Abbott Diagnostics). The CV of the ARCHITECT CA 125 II assay has a CV $<10 \%$. Samples with concentrations higher than $1000 \mathrm{U} / \mathrm{mL}$ were diluted 1:10 using the automated dilution protocol recommended by the manufacturer.

\section{Hs-Tnl assay}

hs-TnI was measured using an Architect i2000 platform (Abbott Diagnostics). There was $\leq 0.1 \%$ cross-reactivity with skeletal troponin I and $\leq 1 \%$ cross-reactivity with cardiac troponin $\mathrm{T}$ and troponin $\mathrm{C}$.

\section{Follow-up and endpoints}

All patients had visits scheduled at regular intervals for 1 to 3 months, with additional visits as required in cases of decompensation or when need of treatment uptitration $^{20}$. Clinical follow-up was subsequently performed by the patient's general practitioner. The composite endpoints, i.e. all-cause mortality or HF-related rehospitalization at 30 days and at 1 year, were the primary endpoints. HF-related rehospitalization was also assessed as a secondary endpoint for both study periods. All-cause death at one year was analyzed as well. Death and hospital admissions were prospectively reviewed using electronic medical records (CP and MD).

\section{Statistical analysis}

Categorical variables were expressed as percentages. Continuous variables were expressed as means (standard deviations [SDs]) or medians [25-75 percentiles] according to their distribution (normal or non-normal). 
Normal distribution was assessed with normal Q-Q plots. Correlation between the different studied biomarkers was performed using Pearson correlation test of log-transformed values of each biomarker. Kaplan-Meier survival curves were plotted for the biomarkers statistically independently associated with the one-year composite end-point and one-year all-cause death and the groups were compared using the Log-Rank test. Univariate Cox regression analyses were performed for all of the endpoints for the four studied biomarkers and also for relevant clinical covariates: age; sex; New York Heart Association functional class; diabetes mellitus; Charlson comorbidity index; Barthel index; and urea, creatinine, hemoglobin, and sodium levels. For HF-related rehospitalization, a competing risk strategy using the Gray method was adopted, considering death as the competing risk. The four studied biomarkers were logtransformed and analyzed per $1 \mathrm{SD}$. Multivariable analyses were also performed using the backward step method, with age, sex, and the variables with statistical significance in the univariate analyses as covariates. Predictive "X"Beta" models were created from Cox regression analysis for the composite endpoint, both at 30 days and at 1 year. The best clinical predictive models without biomarkers were constructed, including age and sex and the variables with statistical significance in the univariate analyses. This approach was used because with such strategy we obtained the better AUC with clinical variables. The area under the curve (AUC) of these "X*Beta" models was then obtained. Next, the biomarkers with statistical significance in univariate analyses were added into the models, and new AUCs were calculated for the predictive biomarkers. AUC were compared by chi-squared test. Statistical analyses were performed with SPSS 15 (SPSS Inc., Chicago, IL, USA) and STATA V.13.0 (College Station, Texas, USA). A two-sided $p<0.05$ was considered significant.

\section{Results}

From February 2014 to November 2016, 522 consecutive patients at the STOP-HF Clinic $(57.1 \%$ women, mean age $82 \pm 8.7$ years, $25 \%$ older than 88 years; mean LVEF $58.8 \%$ \pm 14 ) were included in the STOP-HF-Clinic study. Their clinical characteristics are shown in Table 1. The main etiologies were hypertension (39.7\%), ischemia (28.7\%), and valvular heart disease (13.2\%). Diabetes, anemia, and renal failure were highly prevalent. The mean Barthel index was $70.5 \pm 25.4$, and the mean Charlson comorbidity index was $5.6 \pm 2.2$. Correlations between biomarkers were modest (Additional file 1: Table S1).

\section{Endpoints}

HF-related rehospitalization occurred in 36 patients (6. 9\%) at 30 days and in $137(26.2 \%)$ at 1 year. Death occurred in 13 patients $(2.5 \%)$ at 30 days and in 121 (23. $2 \%)$ at 1 year. Finally, the composite endpoint occurred in 45 patients $(8.6 \%)$ at 30 days and in $201(38.5 \%)$ at 1 year.

At 30 days, diabetes $(p=0.01)$, urea $(p<0.05)$, Barthel index $(p=0.008)$, Charlson comorbidity index $(p<0.001)$, NT-proBNP $(p=0.001)$, and ST2 $(p<0.001)$ were all associated with the primary composite endpoint in the univariate analyses (Table 2). Diabetes $(p=0.007)$, Charlson comorbidity index $(p=0.001)$, NT-proBNP $(p=0.04)$, and ST2 $(p<0.001)$ were associated with HF-related rehospitalization (Additional file 2: Table S2). In multivariable analysis, female sex $(p=0.01)$, Charlson comorbidity index $(p<0.001)$, and ST2 $(p=0.001)$ were the only independent variables associated with the primary composite endpoint (Table 2) and also with HF-related rehospitalization [female sex $(\mathrm{p}=0.04)$, Charlson comorbidity index $(p=0$. $003)$, and ST2 $(p=0.008)$ ] (Additional file 2: Table S2).

Table 3 and Additional file 3: Table S3 show the results of the univariable and multivariable analyses for the composite endpoint and HF-related rehospitalization at one year, respectively. In multivariate analysis, age $(\mathrm{p}=0.003)$, female sex $(p=0.002)$, urea $(p<0.001)$, Charlson comorbidity index $(\mathrm{p}=0.008)$, NT-proBNP $(\mathrm{p}=0.04)$, and ST2 $(\mathrm{p}<0.001)$ remained significantly associated with the composite endpoint (Table 3$)$; only female sex $(\mathrm{p}=0.01)$, urea $(\mathrm{p}<0.001)$, and ST2 $(p=0.001)$ remained significantly associated with HF-related rehospitalization (Additional file 3: Table S3). Figure 1 shows Kaplan-Meier event-free survival curves for the composite end-point relative to circulating levels of ST2 and NTproBNP. Table 4 shows Cox regression analysis results for all-cause death at 1 year. Age $(\mathrm{p}<0.001)$, female sex $(\mathrm{p}=0.008)$, Charlson comorbidity index $(\mathrm{p}=0.001)$, Barthel index $(\mathrm{p}=0.008)$, ST2 ( $\mathrm{p}$ $<0.001)$, and CA125 ( $p=0.003)$ remained independent predictors for all-cause death at 1 year. Figure 2 shows Kaplan-Meier survival curves for all-cause death relative to circulating levels of ST2 and CA125.

\section{Risk prediction}

Risk prediction of the composite endpoint at 30 days for the clinical model, including age, sex, diabetes, urea, Charlson comorbidity index, and Barthel index, revealed an AUC of 0.696 [0.609-0.783]. The addition of NTproBNP and ST2 to the clinical model increased the AUC to 0.750 [0.673-0.827] ( $p=0.02)$ (Fig. 3). Further, reclassification significantly improved [NRI $0.40(0.08-0$. 80), IDI $0.02(-0.002-0.06)]$.

Risk prediction of the composite endpoint at 1 year for the clinical model that included age, sex, NYHA class, urea, hemoglobin, Charlson comorbidity index, and Barthel index revealed an AUC of 0.711 [0.665-0.757]. Addition of the 4 studied biomarkers increased the AUC to 0.736 [0.689-0.783] $(p=0.05)$, and provided significantly better patient reclassification [NRI 0.40 (0.21-0.58), 
Table 1 Clinical characteristics of patients

\begin{tabular}{|c|c|c|c|c|}
\hline & Total & End-point ${ }^{a}$ & No end-point ${ }^{a}$ & $p$-value \\
\hline & $N=522$ & $N=201$ & $N=321$ & \\
\hline Age, years & $82.1 \pm 8.7$ & $84.2 \pm 7.4$ & $80.8 \pm 9.2$ & $<0.001$ \\
\hline Female sex & $298(57.1 \%)$ & $123(61.2 \%)$ & $175(54.5 \%)$ & 0.1 \\
\hline Etiology & & & & 0.3 \\
\hline $\mathrm{IHD}$ & $150(28.7 \%)$ & $45(22.4 \%)$ & $105(32.7 \%)$ & \\
\hline Dilated CM & $9(1.7 \%)$ & $3(1.5 \%)$ & $6(1.9 \%)$ & \\
\hline Hypertensive CM & 207 (39.7\%) & $85(42.3 \%)$ & $122(38.0 \%)$ & \\
\hline Alcoholic CM & $7(1.3 \%)$ & $2(1.0 \%)$ & $5(1.6 \%)$ & \\
\hline Valvular disease & $69(13.2 \%)$ & $31(15.4 \%)$ & $38(11.8 \%)$ & \\
\hline Other & 80 (15.4\%) & 35 (17.4\%) & 45 (20.0\%) & \\
\hline LVEF $^{\mathrm{a}}$ & $55.8 \% \pm 14$ & $56.6 \% \pm 14.2$ & $55.4 \% \pm 13.9$ & 0.4 \\
\hline NYHA functional class & & & & 0.002 \\
\hline । & $8(1.5 \%)$ & $1(0.5 \%)$ & $7(2.2 \%)$ & \\
\hline$\|$ & 198 (37.9\%) & $63(31.3 \%)$ & $136(42.4 \%)$ & \\
\hline III & $306(58.6 \%)$ & $134(66.7 \%)$ & $175(54.5 \%)$ & \\
\hline IV & $6(1.1 \%)$ & $3(1.5 \%)$ & $3(0.9 \%)$ & \\
\hline Diabetes & $274(52.4 \%)$ & $114(56.7 \%)$ & $160(49.8 \%)$ & 0.1 \\
\hline non insulin-dependent & $157(30.1 \%)$ & $64(31.8 \%)$ & $93(29.0 \%)$ & \\
\hline insulin-dependent & 117 (22.4\%) & $50(24.9 \%)$ & 67 (20.9\%) & \\
\hline Hypertension & 468 (89.7\%) & $184(91.5 \%)$ & $284(88.5 \%)$ & 0.3 \\
\hline COPD & $126(24.1 \%)$ & $54(26.9 \%)$ & $72(22.4 \%)$ & 0.3 \\
\hline Renal failure & $411(78.7 \%)$ & $173(86.1 \%)$ & $238(74.1 \%)$ & 0.001 \\
\hline Anaemia & 341 (65.3\%) & $141(70.1 \%)$ & $200(62.3 \%)$ & 0.07 \\
\hline Charlson comorbidity index & $5.6 \pm 2.2$ & $6.1 \pm 2.2$ & $5.3 \pm 2.1$ & $<0.001$ \\
\hline Barthel index & $70.5 \pm 25.4$ & $63.6 \pm 26.5$ & $74.7 \pm 23.7$ & $<0.001$ \\
\hline Core Readmission risk & $26.3 \% \pm 5.2$ & $27.0 \% \pm 5.1$ & $25.8 \% \pm 5.2$ & 0.009 \\
\hline Urea, mg/dL & $90.8 \pm 49.4$ & $107.2 \pm 56.2$ & $80.5 \pm 41.8$ & $<0.001$ \\
\hline Creatinine, mg/dL & $1.4(1.1-2.0)$ & $1.5(1.2-2.1)$ & $1.8(1.0-1.79)$ & $<0.001$ \\
\hline Hemoglobin, g/dL & $11.7 \pm 1.6$ & $11.5 \pm 1.6$ & $11.9 \pm 1.7$ & 0.01 \\
\hline Sodium, mmol/L & $137.3 \pm 3.8$ & $136.9 \pm 4.3$ & $137.5 \pm 3.5$ & 0.08 \\
\hline NTproBNP, ng/L & $2770(1245-5843)$ & $3900(1695-7160)$ & $2170(1025-4635)$ & $<0.001$ \\
\hline $\mathrm{ST} 2, \mathrm{ng} / \mathrm{mL}$ & $42.7(30.9-63.8)$ & $54.6(36.7-79.3)$ & $37.6(29.1-52.3)$ & $<0.001$ \\
\hline $\mathrm{CA} 125^{\mathrm{b}}, \mathrm{U} / \mathrm{ml}$ & $47(22.4-101.1)$ & $57.4(26.7-131.4)$ & $39.2(20.2-90.2)$ & 0.001 \\
\hline $\mathrm{Hs}-\left.\mathrm{Tn}\right|^{\mathrm{b}}, \mathrm{ng} / \mathrm{L}$ & $23.6(12.2-61-2)$ & $28.5(14.8-67.5)$ & $21.9(10.4-57.8)$ & 0.007 \\
\hline
\end{tabular}

${ }^{a}$ Composite end-point all-cause death or heart failure hospitalization at one year ${ }^{\mathrm{b}}$ Available in 466 patients

CM cardiomyopathy, IHD ischemic heart disease, LVEF left ventricular ejection fraction, NYHA New York Heart Association, COPD Chronic obstructive pulmonary disease, CA125 Cancer antigen 125, NTproBNP N-terminal pro-brain natriuretic peptide; $h s$-Tnl high sensitivity troponin I, ST2 Interleukin-1 receptor-like 1

IDI $0.04(0.01-0.08)]$. However, if only NT-proBNP and ST2 (the two biomarkers that were statistically significant in the multivariable analysis) were added to the clinical model, the AUC was virtually the same (0.735 [0.690-0.780]) $\quad(p<0.05$ relative to clinical model), and reclassification improvement remained significant as well [NRI $0.38(0.19-0.55)$, IDI 0.04 (0. 01-0.07)].
Risk prediction for all-cause death at 1 year for the clinical model that included the same covariables revealed an AUC of 0.746 [0.697-0.795]. Addition of the 4 studied biomarkers increased the AUC to 0.789 [0.7390.838 ] $(p=0.01)$. Again, reclassification significantly improved [NRI $0.56(0.36-0.78)$, IDI $0.06 \quad(0.02-0.12]$. However, if only ST2 and CA125 (the two biomarkers that were statistically significant in the multivariable 
Table 2 Cox regression analyses for the 30-day primary endpoint (all-cause death or HF-related rehospitalization)

\begin{tabular}{|c|c|c|c|c|c|c|}
\hline & \multicolumn{6}{|c|}{ 30-day composite endpoint } \\
\hline & \multicolumn{3}{|c|}{ Univariate analysis } & \multicolumn{3}{|c|}{ Multivariate analysis } \\
\hline & $\mathrm{HR}$ & $95 \% \mathrm{Cl}$ & $p$-value & $\mathrm{HR}$ & $95 \% \mathrm{Cl}$ & $p$-value \\
\hline Age & 1.03 & $0.99-1.07$ & 0.2 & - & - & - \\
\hline Female sex & 1.54 & $0.83-2.87$ & 0.2 & 2.28 & $1.19-4.37$ & 0.01 \\
\hline NYHA & 1.08 & $0.63-1.87$ & 0.8 & & & \\
\hline Diabetes & 1.59 & $1.11-2.56$ & 0.01 & - & - & - \\
\hline Charlson comorbidity index & 1.28 & $1.14-1.44$ & $<0.001$ & 1.26 & $1.11-1.43$ & $<0.001$ \\
\hline Barthel index & 0.99 & $0.98-1.00$ & 0.008 & - & - & - \\
\hline Urea & 1.01 & $1.00-1.01$ & $<0.05$ & - & - & - \\
\hline Creatinine & 0.99 & $0.93-1.06$ & 0.7 & & & \\
\hline $\mathrm{Hb}$ & 0.93 & $0.77-1.11$ & 0.4 & & & \\
\hline $\mathrm{Na}$ & 1.05 & $0.97-1.14$ & 0.2 & & & \\
\hline NT-proBNPa & 1.63 & $1.21-2.19$ & 0.001 & - & - & - \\
\hline $\mathrm{ST} 2^{\mathrm{a}}$ & 1.67 & $1.33-2.11$ & $<0.001$ & 1.53 & $1.19-1.97$ & 0.001 \\
\hline $\mathrm{CA} 125^{\mathrm{a}}$ & 1.12 & $0.83-1.51$ & 0.5 & & & \\
\hline $\mathrm{Hs}-\mathrm{Tn} \mathrm{l}^{\mathrm{a}}$ & 1.23 & $0.92-1.63$ & 0.2 & & & \\
\hline
\end{tabular}

aLog-transformed and per 1 SD

CA125 cancer antigen 125, NT-proBNP N-terminal pro-brain natriuretic peptide, hs-Tnl high-sensitivity troponin I, ST2 Interleukin-1 receptor-like 1

analysis) were added to the clinical model, the AUC was practically the same $(0.786[0.736-0.836])(p=0.02$ relative to clinical model), as well as reclassification [NRI 0 . 60 (0.36-0.80), IDI $0.06(0.02-0.11)]$.

\section{Discussion}

This study comprehensively analyzed clinical variables and predictive biomarkers to determine their association with 30-day and 1-year adverse events in comorbid frail elderly patients with mainly HFpEF. In terms of clinical prediction, higher multimorbidity is associated with higher risk of all-cause death and readmission both at 30 days $[16,23]$ and at 1 year [24] despite high-quality multidisciplinary management, especially in the elderly $[24,25]$. In our cohort, the Charlson Index as a measure of comorbidity was associated with both composite endpoints at

Table 3 Cox regression analyses for the 1-year composite endpoint (all-cause death or HF related rehospitalization)

\begin{tabular}{|c|c|c|c|c|c|c|}
\hline & \multicolumn{6}{|c|}{ 1-year composite endpoint } \\
\hline & \multicolumn{3}{|c|}{ Univariate analysis } & \multicolumn{3}{|c|}{ Multivariate analysis } \\
\hline & $\overline{H R}$ & $95 \% \mathrm{Cl}$ & $p$-value & $\overline{H R}$ & $95 \% \mathrm{Cl}$ & $p$-value \\
\hline Age & 1.04 & $1.02-1.06$ & $<0.001$ & 1.03 & $1.01-1.06$ & 0.003 \\
\hline Female sex & 1.28 & $0.96-1.70$ & 0.09 & 1.64 & $1.20-2.23$ & 0.002 \\
\hline NYHA & 1.54 & $1.18-2.01$ & 0.002 & - & - & - \\
\hline Diabetes & 1.16 & $0.98-1.38$ & 0.09 & & & \\
\hline Charlson comorbidity index & 1.15 & $1.08-1.22$ & $<0.001$ & 1.10 & $1.03-1.18$ & 0.008 \\
\hline Barthel index & 0.99 & $0.98-0.99$ & $<0.001$ & - & - & - \\
\hline Urea & 1.01 & $1.01-1.01$ & $<0.001$ & 1.01 & $1.00-1.01$ & $<0.001$ \\
\hline Creatinine & 1.00 & $1.00-1.01$ & 0.3 & - & - & - \\
\hline $\mathrm{Hb}$ & 0.90 & $0.82-0.98$ & 0.01 & - & - & - \\
\hline $\mathrm{Na}$ & 0.97 & $0.93-1.00$ & 0.07 & & & \\
\hline NT-proBNPa & 1.43 & $1.25-1.65$ & $<0.001$ & 1.18 & $1.00-1.38$ & 0.04 \\
\hline $\mathrm{ST} 2^{\mathrm{a}}$ & 1.63 & $1.44-1.85$ & $<0.001$ & 1.41 & $1.21-1.63$ & $<0.001$ \\
\hline $\mathrm{CA} 125^{\mathrm{a}}$ & 1.32 & $1.15-1.52$ & $<0.001$ & - & - & - \\
\hline $\mathrm{Hs}-\left.\mathrm{Tn}\right|^{\mathrm{a}}$ & 1.21 & $1.05-1.39$ & 0.007 & - & - & - \\
\hline
\end{tabular}

aLog-transformed and per 1 SD

CA125 cancer antigen 125, NT-proBNP N-terminal pro-brain natriuretic peptide, $h$ s-Tnl high-sensitivity troponin I, ST2 Interleukin-1 receptor-like 1 

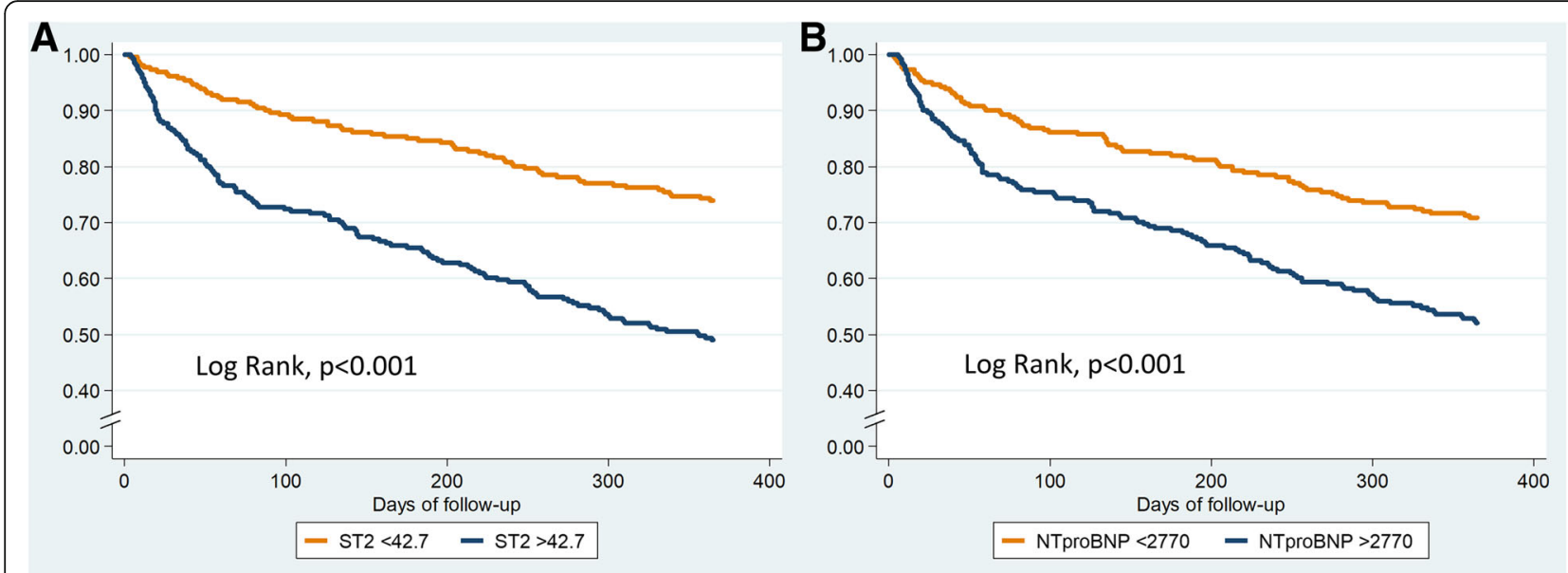

Fig. 1 Kaplan-Meier event-free survival curves for the composite end-point relative to serum levels of ST2 (a) and NTproBNP (b) above or below the median

30 days and 1 year and also with HF-related rehospitalization at 30 days. The prognostic role of the Charlson comorbidity index has been described previously [5, 26], but in our study, it remained independently associated with outcome despite the inclusion of strong predictive biomarker variables. Remarkably, both the primary composite endpoint and the secondary endpoint of 30-day HF rehospitalization were only associated with female sex, Charlson comorbidity index, and ST2.

Notably, predictive biomarkers showed value beyond that of clinical risk factors, since circulating biomarkers have the potential to predict the risk of recurrent hospitalization or death [27, 28]. In our elderly cohort, ST2 emerged as the strongest predictor in both univariate and multivariate analysis for all of the endpoints, which is in line with recent reports [29]. In our study, NT-proBNP, hs-TnI, and CA-125 lost their statistical significance when ST2 and clinical variables were included in the model.

The addiction of circulating ST2 to our model was based on its value as a surrogate of inflammation, stretch, and extracellular matrix status as well as on the observation that the ST2 concentration is not affected by age, renal function [30], or body mass index [31].

Table 4 Cox regression analyses for 1-year all-cause death

\begin{tabular}{|c|c|c|c|c|c|c|}
\hline & \multicolumn{6}{|c|}{ 1-year all-cause death } \\
\hline & \multicolumn{3}{|c|}{ Univariate } & \multicolumn{3}{|c|}{ Multivariate } \\
\hline & $\mathrm{HR}$ & $95 \% \mathrm{Cl}$ & $p$-value & $\mathrm{HR}$ & $95 \% \mathrm{Cl}$ & $p$-value \\
\hline Age & 1.08 & $1.05-1.11$ & $<0.001$ & 1.08 & $1.05-1.12$ & $<0.001$ \\
\hline Female Sex & 1.28 & $0.89-1.85$ & 0.2 & 1.73 & $1.16-2.59$ & 0.008 \\
\hline NYHA & 2.01 & $1.40-2.90$ & $<0.001$ & - & - & - \\
\hline Diabetes & 1.03 & $0.83-1.29$ & 0.8 & & & \\
\hline Charlson comorbidity index & 1.15 & $1.05-1.26$ & 0.002 & 1.17 & $1.06-1.28$ & 0.001 \\
\hline Barthel index & 0.98 & $0.97-0.99$ & $<0.001$ & 0.99 & $0.98-1.00$ & 0.008 \\
\hline Urea & 1.01 & $1.00-1.01$ & $<0.001$ & - & - & - \\
\hline Creatinine & 1.00 & $1.00-1.01$ & 0.4 & & & \\
\hline $\mathrm{Hb}$ & 0.87 & $0.77-0.97$ & 0.02 & - & - & - \\
\hline $\mathrm{Na}$ & 0.97 & $0.93-1.02$ & 0.2 & & & \\
\hline NT-proBNPa & 1.65 & $1.37-1.98$ & $<0.001$ & - & - & - \\
\hline $\mathrm{ST} 2^{\mathrm{a}}$ & 1.86 & $1.59-2.18$ & $<0.001$ & 1.45 & $1.21-1.74$ & $<0.001$ \\
\hline $\mathrm{CA} 125^{\mathrm{a}}$ & 1.58 & $1.33-1.89$ & $<0.001$ & 1.35 & $1.11-1.65$ & 0.003 \\
\hline$H s-\left.T n\right|^{a}$ & 1.29 & $1.09-1.52$ & 0.003 & - & - & - \\
\hline
\end{tabular}

aLog-transformed and per 1 SD

CA125 cancer antigen 125, NT-proBNP N-terminal pro-brain natriuretic peptide, $h$ s-Tnl high-sensitivity troponin I, ST2 Interleukin-1 receptor-like 1 

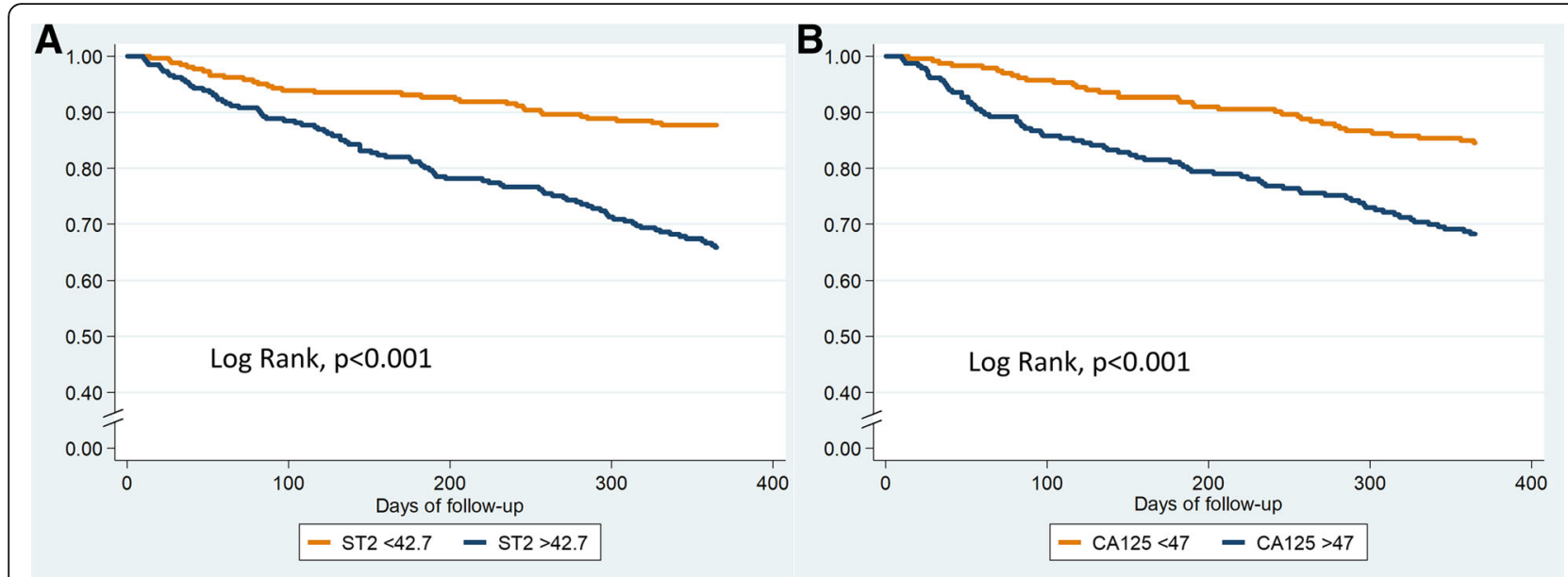

Fig. 2 Kaplan-Meier survival curves for all-cause death relative to serum levels of ST2 (a) and CA125 (b) above or below the median

These characteristics are highly desirable in a marker that will be used in an elderly population with much comorbidity. Our current data is in line with the notion that ST2 reflects systemic inflammatory disease involving multiple organ systems [32] and that it is related to HFpEF development and to the proinflammatory and profibrotic state [33]. Indeed, it was recently postulated that ST2 is the new "gold standard" for HF prognostication and monitoring [29].

NT-proBNP, a marker of myocardial stretch and neurohormonal activation, is the only biomarker that is currently included in HF guidelines [34] and it has shown

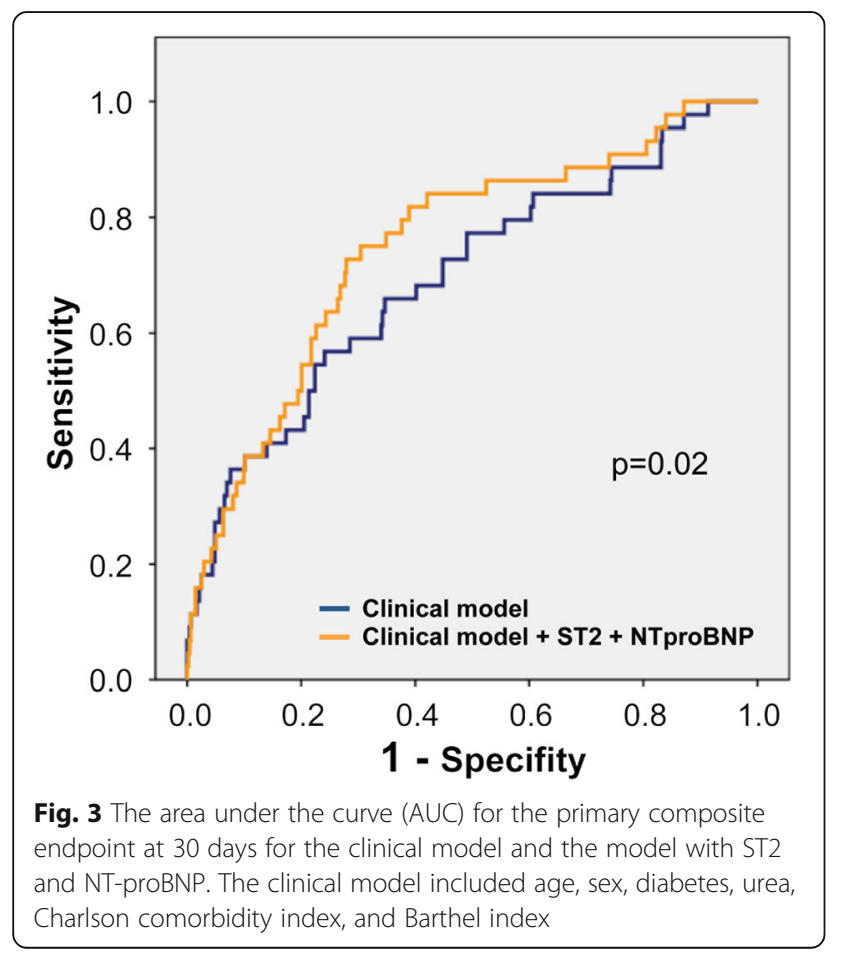

incremental diagnostic and prognostic value in $\mathrm{HFrEF}$ and HFpEF [35, 36]. However, certain conditions, which are highly prevalent in our patient population, can make it difficult to interpret this versatile biomarker. These conditions include the presence of underlying structural cardiopulmonary diseases (chronic obstructive disease, pulmonary hypertension), anemia, advanced age, atrial fibrillation, female sex, and renal failure, all of which result in high levels of NT-proBNP. By contrast, obesity is well recognized as a condition that reduces circulating NT-proBNP levels. In the current study, NT-proBNP lost its discriminative value: it showed a significant association with the composite endpoint at 1 year, but no significant association with HF-related rehospitalization or 30-day outcomes.

Hs-TnI was included in our analysis because it is generally accepted that troponins offer valuable information for risk stratification during acute decompensation in patients with HFpEF [37]. High levels of hs-TnI have been associated with worse in-hospital, 30-day, and 1year survival and with longer length of stay and more readmission risk within 30 days [37]. Nevertheless, our data showed that when a multi-biomarker panel was included in the multivariable analysis, hs-TnI lost its predictive value in this high-risk population. Further, as is the case for natriuretic peptides, troponins are strongly influenced by age, history of diabetes mellitus, and lower estimated glomerular filtration rate [38].

CA125 is an interesting biomarker. It has traditionally been linked to ovarian neoplasm, but more recently it has been associated with tissue congestion. Núñez et al. [39] found that CA125 as measured during acute HF hospitalization is an independent predictor of mortality [39]. In our cohort, CA125 also emerged as an independent prognosticator of all-cause death at 1 year, but it did not provide additional value to the primary endpoint at 
the 30-day or 1-year follow-up. In the STOP-HF Clinic [20] special attention has been paid to treatment of heart congestion; indeed, $100 \%$ of the patients were on diuretic therapy, and more than 300 infusions of IV furosemide were administered during the first month of follow-up. The neutral effect of CA125 regarding the composite endpoints may partly be explained by the fact that its assessment was not performed during the acute phase of HF decompensation, where prior works pointed out its value as marker of fluid overload. However, it is remarkable that CA125 remained independently associated with 1-year allcause mortality regardless it was not measured during the maximum wet phase. Further studies are needed to better understand the value of CA125 in HF.

Historically, it has been much more difficult to estimate the risk of HF hospitalization than to estimate the risk of death [40]. The high rate of readmissions within 30 days post-discharge and during the following year in ageing and comorbid patients highlights the urgency of establishing predictive models with acceptable performance in such patients to guide clinical management and decision making. To our knowledge, the predictive models based on biomarkers that were developed in this study are the first that can be used in this comorbid fragile elderly population with HF. The BIOSTAT-CHF study recently developed and validated three risk models that included biomarkers (natriuretic peptides) to predict all-cause mortality, HF-related hospitalization, and the composite endpoint in a cohort of patients with worsening HF. The patients included in their cohort were a mixture of in-hospital and ambulatory patients with a mean age of 69 years, 27\% female, and mean LVEF of $31 \%$ [41]; in other words, theirs was a relatively young and mainly male HFrEF population that was quite different from our study cohort. These researchers obtained C-statistic values of $0.73,0.69$, and 0.71 for allcause mortality, HF-related hospitalization, and the composite endpoint, respectively; these were lower than those reported in the present study, and their follow-up time was 21 months. No short-term predictions were performed.

\section{Limitations}

Our study was an observational single-center study with a cohort of elderly patients with comorbidities that mainly had HF of hypertensive etiology and preserved ejection fraction. As such, our results cannot be extrapolated to a global HF population. In fact, the absence of predictive models in such patients may be a strength rather than a limitation given the growing number of $\mathrm{HF}$ patients with this phenotype.

The biomarker selection was based on previously described predictive value of the biomarkers, on other information about HF pathways, and on its availability for use in routine clinical practice. The optimal panel of biomarkers remains debatable. One major limitation of this study is the absence of a validation cohort. However, we have not been able to identify a post-discharge cohort with similar clinical characteristics with available multi-biomarker data.

\section{Conclusions}

In a comorbid fragile elderly population with mainly HFpEF, ST2 outperformed NT-proBNP for risk prediction of the composite primary endpoint (all-cause mortality or HF-related rehospitalization). Regarding 1year all-cause mortality, ST2 together with CA125 were independently associated with higher risk and the addition of both biomarkers improved the discriminative accuracy of clinical data. ST2 is a three-in-one biomarker as a surrogate of inflammation, stretch, and extracellular matrix remodeling, and it may be the preferred biomarker for short- and long-term prediction in a high-risk HF population like ours. Comorbidities, as represented by the Charlson comorbidity index, also strongly impacted these patients' outcomes. Further studies in similar populations are needed to confirm our results.

\section{Additional files}

Additional file 1: Table S1. Correlations between studied biomarkers. Correlation between the different studied biomarkers was performed using Pearson correlation test of log-transformed values of each biomarker. (DOCX 25 kb)

Additional file 2: Table S2. Cox regression analyses for 30-day rehospitalization. A competing risk strategy using the Gray method was adopted, considering death as the competing risk in both univariate and multivariate Cox regression analyses. (DOCX $27 \mathrm{~kb}$ )

Additional file 3: Table S3. Cox regression analyses for 1-year HFrelated hospitalization. A competing risk strategy using the Gray method was adopted, considering death as the competing risk in both univariate and multivariate Cox regression analyses. (DOCX $27 \mathrm{~kb}$ )

\section{Abbreviations}

CA125: Cancer antigen 125; HF: Heart failure; HFpEF: Heart failure with preserved ejection fraction; Hs-Tnl: High-sensitivity troponin l; NT-proBNP: Nterminal pro-brain natriuretic peptide; ST2: Interleukin-1 receptor-like 1; STOPHF-Clinic: STructured multidisciplinary outpatient clinic for Old and frail Postdischarge patients hospitalized for HF

\section{Acknowledgements}

We thank Beatriz González, Roser Cabanes, Margarita Rodríguez, and Carmen Rivas, all of whom are nurses in the HF Unit, for expert help with data collection and for their invaluable work in the unit.

\section{Funding}

AB-G was supported by grants from the Ministerio de Educación y Ciencia (SAF2014-59892), Fundació La MARATÓ de TV3 (201502, 201516), CIBER Cardiovascular (CB16/11/00403), and AdvanceCat 2014-2020. JL and SR by grant from Fondo de Investigación Sanitaria, Instituto de Salud Carlos III (FIS PI14/01682)

JN was supported by grants from CIBER Cardiovascular (CB16/11/00420) and PIE15/00013. 
Any of these entities had a role in the design, methods, subject recruitment, data collections, analysis and preparation of paper.

\section{Availability of data and materials}

Data and material of the study are available from the corresponding author on reasonable request.

\section{Authors' contributions}

CP: acquisition of subjects and/or data, drafting the manuscript, revision for important intellectual content and approval of the final manuscript. MD: acquisition of subjects and/or data, revision for important intellectual content and approval of the final manuscript. RN: acquisition of subjects and/or data, revision for important intellectual content and approval of the final manuscript. JL: study concept and design, statistical analyses, analysis and interpretation of data, drafting the manuscript, revision for important intellectual content and approval of the final manuscript. JN: analysis and interpretation of data, drafting the manuscript, revision for important intellectual content and approval of the final manuscript. JB: acquisition of subjects and/or data, revision for important intellectual content and approval of the final manuscript. PM: acquisition of subjects and/or data, revision for important intellectual content and approval of the final manuscript. MA: acquisition of subjects and/or data, revision for important intellectual content and approval of the final manuscript. JS: acquisition of subjects and/ or data, revision for important intellectual content and approval of the final manuscript. GC: statistical analyses and interpretation of data, revision for important intellectual content and approval of the final manuscript. MCP. acquisition of subjects and/or data, revision for important intellectual content and approval of the final manuscript. SR: analysis and interpretation of data, revision for important intellectual content and approval of the final manuscript. JT: analysis and interpretation of data, revision for important intellectual content and approval of the final manuscript. AB-G: study concept and design, analysis and interpretation of data, drafting the manuscript, revision for important intellectual content and approval of the final manuscript.

\section{Ethics approval and consent to participate}

All participants provided written informed consent, and the study was approved by the local ethics committee (Comité Ėtic de la Investigació de I'Hospital Universitari Germans Trias i Pujol; EO-10-076). All study procedures were performed in accordance with the ethical standards outlined in the Helsinki Declaration of 1975 as revised in 2013

\section{Competing interests}

$J L$ received lecture honoraria from Roche Diagnostics and reports a relationship with Critical Diagnostics.

JN received board membership fees and travel expenses from Novartis, Roche Diagnostics, Abbott, Rovi, and Vifor.

$A B-G$ received board membership fees and travel expenses from Novartis and Roche Diagnostics and reports a relationship with Critical Diagnostics. The rest of the authors have no disclosures to mention.

\section{Publisher's Note}

Springer Nature remains neutral with regard to jurisdictional claims in published maps and institutional affiliations.

\footnotetext{
Author details

${ }^{1}$ Servei de Medicina Interna i Unitat de Geriatria d'Aguts, Hospital Universitari Germans Trias i Pujol, Badalona, Barcelona, Spain. ${ }^{2}$ Servei de Cardiologia i Unitat d'Insuficiència Cardíaca, Hospital Universitari Germans Trias i Pujol, Badalona, Barcelona, Spain. ${ }^{3}$ Servei de Bioquímica i Anàlisis clíniques, Hospital Universitari Germans Trias i Pujol, Badalona, Barcelona, Spain. ${ }^{4}$ Department de Medicina, Universitat Autònoma de Barcelona, Barcelona, Spain. ${ }^{5} \mathrm{CIBERCV}$, Instituto de Salud Carlos III, Madrid, Spain. ${ }^{6}$ Cardiology Department, Hospital Clínico Universitario, INCLIVA Valencia, Valencia, Spain. ${ }^{7}$ Departamento de Medicina, Universidad de Valencia, Valencia, Spain. ${ }^{8}$ ICREC Research Program, Germans Trias i Pujol Health Science Research Institute, Badalona, Spain.
}

Received: 25 January 2018 Accepted: 3 May 2018

Published online: 09 May 2018

\section{References}

1. Fonarow GC. Epidemiology and risk stratification in acute heart failure. Am Heart J. 2008;155:200-7.

2. Dharmarajan K, Hsieh AF, Lin Z, Bueno H, Ross JS, Horwitz LI, et al. Diagnoses and timing of 30-day readmissions after hospitalization for heart failure, acute myocardial infarction, or pneumonia. JAMA. 2013;309:355-63.

3. Dharmarajan K, Krumholz HM. Strategies to reduce 30-day readmissions in older patients hospitalized with heart failure and acute myocardial infarction. Curr Geriatr Rep. 2014;3:306-15.

4. Desai AS, Stevenson LW. Rehospitalization for heart failure: predict or prevent? Circulation. 2012;126:501-6.

5. Arora S, Lahewala S, Hassan Virk HU, Setareh-Shenas S, Patel P, Kumar V, et al. Etiologies, trends, and predictors of 30-day readmissions in patients with diastolic heart failure. Am J Cardiol. 2017;120:616-24.

6. Chioncel O, Mebazaa A, Harjola VP, Coats AJ, Piepoli MF, Crespo-Leiro MG, et al. Clinical phenotypes and outcome of patients hospitalized for acute heart failure: the ESC heart failure long-term registry. Eur J Heart Fail. 2017; 19:1242-54

7. Writing Group Members, Mozaffarian D, Benjamin EJ, Go AS, Arnett DK, Blaha MJ, Cushman M, et al. American Heart Association Statistics Committee; Stroke Statistics Subcommittee. Heart disease and and stroke statistics-2016 update: a report from the American Heart Association. Circulation 2016:133:447-54.

8. Farré $N$, Vela $E$, Clèries $M$, Bustins $M$, Cainzos-Achirica $M$, Enjuanes $C$, et al. Medical resource use and expenditure in patients with chronic heart failure: a population-based analysis of 88195 patients. Eur J Heart Fail. 2016;18:1132-40

9. Ambrosy AP, Fonarow GC, Butler J, Chioncel O, Greene SJ, Vaduganathan M Nodari $S$, et al. The global hearth and economic burden of hospitalizations for heart failure: lessons learned from hospitalized heart failure registries. J Am Coll Cardiol. 2014;63:1123-33.

10. McMurray JJ, Pfeffer MA. Heart failure Lancet. 2005:365:1877-89.

11. Curtis LH, Whellan DJ, Hammill BG, Hernandez AF, Anstrom KJ, Shea AM, et al. Incidence and prevalence of heart failure in elderly persons, 1994-2003. Arch Intern Med. 2008;168:418-24.

12. Wong CY, Chaudhry SI, Desai MM, Krumholz HM. Trends in comorbidity, disability, and polypharmacy in heart failure. Am J Med. 2011;124:136-43.

13. Van Riet EE, Hoes AW, Wagenaar KP, Limburg A, Landman MA, Rutten FH. Epidemiology of heart failure: the prevalence of heart failure and ventricular dysfunction in older adults over time. A systematic review. Eur J Heart Fail. 2016;18:242-52.

14. Lazzarini V, Mentz RJ, Fiuzat M, Metra M, O'Connor CM. Heart failure in elderly patients: distinctive features and unresolved issues. Eur J Heart Fail. 2013;15:717-23.

15. Comin-colet J, Verdu-Rotellar JM, Vela E, Clèries M, Bustins M, Mendoza L et al. working group of the Integrated Program for Heart Failure Management of the Barcelona Litoral Mar Integrated Health Care Area, Spain. Efficacy of an integrated hospital-primary care program for heart failure: a population-based analysis of 56,742 patients. Rev Esp Cardiol. 2014; 67:283-93.

16. Davis JD, Olsen MA, Bommarito K, LaRue SJ, Saeed M, Rich MW, et al. Allpayer analysis of heart failure hospitalization 30-day readmission: comorbidities matter. Am J Med. 2017;130:93.e9-93.e28.

17. Steyerberg EW. Applications of prediction models. In: Clinical prediction models: a practical approach to Develeopment validation and updating. 1st ed. New York: Springer; 2009.

18. Dunbar-Yaffe R, Stitt A, Lee JJ, Mohamed S, Lee DS. Assessing risk and preventing 30-day readmissions in decompensated heart failure: opportunity to intervene? Curr Heart Fail Rep. 2015;12:309-17.

19. Lupón J, de Antonio M, Galán A, Vila J, Zamora E, Urrutia A, et al. Combined use of the novel biomarkers high-sensitivity troponin $\mathrm{T}$ and ST2 for heart failure risk stratification vs conventional assessment. Mayo Clin Proc. 2013:88:234-43.

20. Correale M, Monaco I, Brunetti ND, Di Biase M, Metra M, Nodari S, et al. Master Program Students on Drug Development for Heart Failure Redefining biomarkers in heart failure. Heart Fail Rev. 2018;23:237-53.

21. Pacho $C$, Domingo $M$, Nuñez $R$, Lupón J, Moliner $P$, de Antonio $M$, González B, et al. Early Postdischarge STOP-HF-Clinic reduces 30-day 
readmissions in old and frail patients with heart failure. Rev Esp Cardiol (Engl Ed). 2017;70:631-8.

22. Ho KK, Anderson JM, Kannel WB, Grossman W, Levy D. Survival after the onset of congestive heart failure in Framingham HEart study subjects. Circulation. 1993;88:107-15.

23. Wiley JF, Chan YK, Ahamed Y, Ball J, Carrington MJ, Riegel B, et al. Multimorbidity and the risk of all-cause 30-day readmission in the setting of multidisciplinary Management of Chronic Heart Failure: a retrospective analysis of 830 hospitalized patients in Australia. J Cardiovasc Nurs. 2017 https://doi.org/10.1097/JCN.0000000000000391. [Epub ahead of print]

24. Rosamond W, Flegal K, Furie K, Go A, Greenlund K, Haase N, et al; American Heart Association Statistics Committee and Stroke Statistics Subcommittee. Heart disease and stroke statistics - 2008 an update: a report from the American Heart Association statistics Commitee and stroke statistics Subcommitee. Circulation 2008;117:e25-e146.

25. Giamouzis G, Kalogeropoulos A, Georgiopoulou V, Laskar S, Smith AL, Dunbar $\mathrm{S}$, et al. Hospitalization epidemic in patients with heart failure: risk factors, risk prediction, knowledge gaps, and future directions. J Card Fail. 2011:17:54-75.

26. Au AG, McAlister FA, Bakal JA, Ezekowitz J, Kaul P, van Walraven C. Predicting the risk of unplanned readmission or death within 30 days of discharge after a heart failure hospitalization. Am Heart J. 2012;164:365-72.

27. Demissei BG, Postmus D, Cleland JG, O'Connor CM, Metra M, Ponikowski P, et al. Plasma biomarkers to predict or rule out early post-discharge events after hospitalization for acute heart failure. Eur J Heart Fail. 2017;19:728-38

28. Bayes-Genis A, Núñez J, Núñez E, Martínez JB, Ferrer MP, de Antonio M, et al. Multi-biomarker profiling and recurrent hospitalizations in heart failure. Front Cardiovasc Med. 2016;3:37.

29. Bayes-Genis A, Núñez J, Lupón J. Soluble ST2 for prognosis and monitoring in heart failure: the new gold standard? J Am Coll Cardiol. 2017;70:2389-92.

30. Maisel AS, Richards M, Pascual-Figal D, Mueller C. Serial ST2 testing in hospitalized patients with acute heart failure. Am J Cardiol. 2015;115(7 Suppl):32B-7B.

31. Chow SL, Maisel AS, Anand I, Bozkurt B, de Boer RA, Felker GM, et al; American Heart Association Clinical Pharmacology Committee of the Council on Clinical Cardiology; Council on Basic Cardiovascular Sciences; Council on Cardiovascular Disease in the Young; Council on Cardiovascular and Stroke Nursing; Council on Cardiopulmonary, Critical Care, Perioperative and Resuscitation; Council on Epidemiology and Prevention; Council on Functional Genomics and Translational Biology: and Council on Quality of Care and Outcomes Research. Research role of biomarkers for the prevention, assessment, and Management of Heart Failure: a scientific statement from the American Heart Association. Circulation 2017;135:e1054-e1091.

32. Bayes-Genis A, Januzzi IL, Gaggin HK, de Antonio M, Motiwala SR, Zamora E, et al. ST2 pathogenetic profile in ambulatory heart failure patients. J Card Fail. 2015;21:355-61.

33. Zile MR, Baicu CF, Ikonomidis JS, Stroud RE, Nietert PJ, Bradshaw AD, et al. Myocardial stiffness in patients with heart failure and a preserved ejection fraction: contributions of collagen and titin. Circulation. 2015;131:1247-59.

34. Yancy CW, Jessup M, Bozkurt B, Butler J, Casey DE Jr, Colvin MM, et al. 2017 ACC/AHA/HFSA focused update of the 2013 ACCF/AHA guideline for the Management of Heart Failure: a report of the American College of Cardiology/American Heart Association task force on clinical practice guidelines and the Heart Failure Society of America. J Am Coll Cardiol. 2017; 70:776-803.

35. Mallick A, Januzzi JL Jr. Biomarkers in acute heart failure. Rev Esp Cardiol. 2015;68:514-25

36. Kang S, Park JJ, Choi D, Yoon CH, Oh IY, Kang SM, et al. KorHF Registry. Prognostic value of NT-proBNP in heart failure with preserved versus reduced EF. Heart 2015;101:1881-8.

37. Pandey A, Golwala H, Sheng S, DeVore AD, Hernandez AF, Bhatt DL, et al. Factors associated with and prognostic implications of cardiac troponin elevation in decompensated heart failure with preserved ejection fraction: findings from the American Heart Association get with the guidelines-heart failure program. JAMA Cardiol. 2017;2:136-45.

38. Jhund PS, Claggett BL, Voors AA, Zile MR, Packer M, Pieske BM, et al. PARAMOUNT Investigators. Elevation in high-sensitivity troponin $\mathrm{T}$ in heart failure and preserved ejection fraction and influence of treatment with the angiotensin receptor Neprilysin inhibitor LCZ696. Circ Heart Fail. 2014;7:953-9.
39. Núñez J, Núñez E, Consuegra L, Sanchis J, Bodí V, Martínez-Brotons A, et al. Carbohydrate antigen 125: an emerging prognostic risk factor in acute heart failure? Heart. 2007;93:716-21.

40. Ouwerkerk W, Voors AA, Zwinderman AH. Factors influencing the predictive power of models for predicting mortality and/or heart failure hospitalization inpatients with heart failure. JACC Heart Fail. 2014;2:429-36.

41. Voors AA, Ouwerkerk W, Zannad F, van Veldhuisen DJ, Samani NJ, Ponikowski $P$, et al. Development and validation of multivariable models to predict mortality and hospitalization in patients with heart failure. Eur J Heart Fail. 2017;19:627-34.

\section{Ready to submit your research? Choose BMC and benefit from:}

- fast, convenient online submission

- thorough peer review by experienced researchers in your field

- rapid publication on acceptance

- support for research data, including large and complex data types

- gold Open Access which fosters wider collaboration and increased citations

- maximum visibility for your research: over $100 \mathrm{M}$ website views per year

At BMC, research is always in progress.

Learn more biomedcentral.com/submissions 